Saudi Journal of Oral and Dental Research

Abbreviated Key Title: Saudi J Oral Dent Res

ISSN 2518-1300 (Print) |ISSN 2518-1297 (Online)

Scholars Middle East Publishers, Dubai, United Arab Emirates

Journal homepage: https://saudijournals.com/sjodr

\title{
Systematic Review Protocol on: Role of Arthroscopic Lysis and Lavage in Management of Internally Deranged Temporomandibular Joint
}

\author{
Abugharsa $\mathrm{W}^{1^{*}}$, Chong $\mathrm{J}^{2}$, Abdullah $\mathrm{N}^{2}$, Rokhani $\mathrm{F}^{2}$ \\ ${ }^{1}$ Postgraduate Student, Universiti Sains Islam Malaysia Dental College, Kuala Lumpur, Malaysia \\ ${ }^{2}$ Senior Lecture, Universiti Sains Islam Malaysia Dental College, Kuala Lumpur, Malaysia
}

DOI: $\underline{10.36348 / \text { sjodr.2020.v05i03.010 }}$

| Received: 19.03.2020 | Accepted: 26.03.2020 | Published: 27.03.2020

*Corresponding author: Waheeb Abugharsa

\section{Abstract}

The aim of this systematic review is to determine the effectiveness of arthroscopic lysis and lavage as a diagnostic and therapeutic tool for management of internal derangement of temporomandibular joint. Method: A systematic review will be conducted according to the guidance of Cochrane Collaboration and the Preferred Reporting Items for Systematic Reviews and Meta-Analyses (PRISMA) statement. Search strategy will be tailored according to Population, Intervention, Comparison, Outcome and Study design (PICOS) approach. Ovid Medline, CINAHL Complete, ScienceDirect, Web of Science, Cochrane centre register for clinical trials and Pub-med data bases will be searched from1975 to 2020. Truncation, Boolean and Proximity operators will be applied as appropriate. Cochrane's tool for risk of bias assessment will be used for randomised trials and for non-randomised studies, Newcastle Ottawa Scale will be used. Narrative synthesis will be conducted for all the included studies and if an adequate data were available, a meta-analysis will be conducted. Significance: The study will systematically assess the therapeutic potential of arthroscopic lysis and lavage and define its position in the ladder of management of internal derangement of temporomandibular joint in comparison to other lines of treatment. It will also investigate the diagnostic limit of temporomandibular joint arthroscopy in assessment of disc status and its ability to detect adhesions and perforations in comparison to magnetic resonance imaging.

Keywords: Temporomandibular Joint, Arthroscopy, Magnetic Resonance Imaging.

PROSPERO registration number: CRD42019133126.

Copyright @ 2020: This is an open-access article distributed under the terms of the Creative Commons Attribution license which permits unrestricted use, distribution, and reproduction in any medium for non-commercial use (NonCommercial, or CC-BY-NC) provided the original author and source are credited.

\section{INTRODUCTION}

Internal derangement (ID) is a pathological condition of disk displacement of the temporomandibular joint (TMJ) [1]. ID of TMJ is one of the most common forms of temporomandibular disorders (TMD) [2]. It is estimated that $30 \%$ of the population has been reported with some form of ID of TMJ. However, only a small percentage has symptoms that are severe enough to limit function [3].

In 1975, Onishi first reported the use of the arthroscope in TMJ for diagnostic purposes, then later, Sanders, Murakami and McCain described different techniques of therapeutic application of TMJ arthroscopy [4].

Treatment options range from nonsurgical (reversible) therapies to surgical (irreversible) therapies. Surgical treatment is classified as minimally invasive procedures (arthroscopy and arthrocentesis) or open procedures (discoplasty and discectomy) [5].
Arthroscopic lysis and lavage (ALL) is the simplest form of TMJ arthroscopy and can be used in a single or double puncture technique [6]. The effect of ALL lies primarily in the irrigation of the joint cavity, washout of inflammatory cytokines, mobilization of the articular disk, stretching of the capsule and lysis of intra articular adhesions [7].

Currently, there are several modalities to diagnose ID, magnetic resonance imaging (MRI) and arthroscopy represent the most effective and reliable methods between the contemporary techniques. Moreover, they are characterized by being generally safe when compared to old techniques [8].

MRI represents the best method for studying clinically diseased joints [9]. It is the optimal method of imaging the soft tissues and hard tissue composition changes of the TMJ as the technique is non-invasive and has a high diagnostic accuracy for both intra and extra articular changes [10]. 
TMJ arthroscopy has the distinct advantage of allowing direct visualization of the TMJ and fulfils not only a therapeutic role but also a diagnostic one, where these intra-articular findings could warrant further surgical intervention or change of treatment plan [11, 12] with high sensitivity and specificity [5].

The main pathological changes found during arthroscopic procedures are adhesions, synovitis, chondromalacia, and disc displacement. The latter is assessed by the roofing which is the degree of coverage of the condyle by the articular disc, which is related to the degree of disc displacement [5].

Roofing is defined as coverage of the condyle by the disc when the condyle is in a seated and open position during arthroscopy. It is graded according to the posterior band of the disc and its position relative to the articular eminence $[4,13,14]$. Dis position, degree of disc displacement as well as the ability of the disc to reduce, can be fairly assessed under direct observation by the arthroscope [15].
Giving the above-mentioned information, we propose a systematic and reproducible strategy to investigate the therapeutic potential of ALL and its diagnostic capacity in management of ID of TMJ.

\section{METHODS AND ANALYSIS}

This systematic review will be conducted in accordance with the Cochrane Collaboration [16] and is reported according to the Preferred Reporting Items for Systematic Reviews and Meta-Analyses (PRISMA) statement [17].

\section{Search Strategy}

The search strategy was designed to capture the maximum number of citations according to the Population, Intervention, Comparison, Outcome and Study Design (PICOS) approach (Table-1) [18, 19]. We elaborated the guiding question which is "How valuable is TMJ arthroscopy in the management of internal derangement of the temporomandibular joint?"

Table-1: The inclusion and exclusion criteria based on the PICOS strategy

\begin{tabular}{|l|l|l|}
\hline $\begin{array}{l}\text { PICOS } \\
\text { Strategy }\end{array}$ & Inclusion Criteria & Exclusion Criteria \\
\hline Population & $\begin{array}{l}\text { All patients diagnosed with ID of the TMJ regardless of the aetiology. No age } \\
\text { restriction. }\end{array}$ & Animal studies \\
\hline Intervention & $\begin{array}{l}\text { All trials which used TMJ arthroscopy in the management of ID of TMJ will be } \\
\text { included. }\end{array}$ & $\begin{array}{l}\text { Reviews } \\
\text { for reference only). }\end{array}$ \\
\hline Comparison & $\begin{array}{l}\text { Therapeutic: Other standard lines of surgical treatment. } \\
\text { Diagnostic: Magnetic resonance imaging (MRI). }\end{array}$ & XXX \\
\hline $\begin{array}{l}\text { Outcome of } \\
\text { Interest }\end{array}$ & $\begin{array}{l}\text { Primary outcome: } \\
\text { Pain reduction and improvement of mouth opening. } \\
\text { Secondary outcome: } \\
\text { Assessment of disc status and detection of adhesions and perforations. }\end{array}$ & $\begin{array}{l}\text { Studies that do not } \\
\text { report the outcomes }\end{array}$ \\
\hline Studies Type & $\begin{array}{l}\text { All published clinical studies of ID of TMJ managed with arthroscopy will be } \\
\text { considered for inclusion. However, only studies reported in English and done on } \\
\text { humans will be included. }\end{array}$ & XXX \\
\hline
\end{tabular}

All studies are retrieved using six databases: Medline (via Ovid), CINAHL Complete, Web of Science, Science Direct, Cochrane Central Register of Controlled Trials (CENTRAL) and Pubmed. We will search the literature of the last 4 decades as the first paper describing TMJ arthroscopy was published in 1975 by Onishi [4]. We only restrict the language to English as most of the high standard publications are in English and we have no capacity to translate other language to English. To ensure that most of the relevant studies will be identified, we will conduct bibliographic search from the eligible studies as well as other published systematic reviews related.

Each reference obtained from the searches are exported and merged into Mendeley version 1.19.5. All duplicates are identified and inspected individually to prevent the error of deleting relevant studies.

In the process of searching the relevant studies, we have listed down the keywords of the study which are "temporomandibular joint", "arthroscopy" and "magnetic resonance imaging". In terms of the diagnostic value of the TMJ arthroscopy, we also listed down keywords such as "diagnosis", "sensitivity", "specificity" and "validity". Similarly, for the therapeutic purposes we used keywords such as "surgical procedures" and the keywords for clinical trial study design [20]. We verified the keywords and other terms related using the MeSH terms. Synonyms and possible abbreviations of the keywords were also listed. We used proper truncation wherever appropriate and combined the search terms using Boolean operators "AND" and "OR".

Following this, a search strategy such as (temporomandibular adj3 (joint OR disease OR disorder) AND (diagnosis) AND (sensitivity OR specificity OR validity) AND (magnetic resonance imaging OR MRI) was used to identified studies using TMJ arthroscopy as a diagnostic tool. In order to locate studies which used TMJ arthroscopy as intervention, we 
used similar search strategy as above and crossed the search with (randomised control trial OR controlled clinical trial OR clinical trial OR trial).

\section{Study Selection Criteria}

The eligibility criteria using the PICOS strategy is provided in table 1 . Since the term management includes the process of investigation as well as treatment of the condition, we include all studies published that have used TMJ arthroscopy either as a diagnostic tool to identify ID or as a treatment modality for ID of the TMJ.

The outcomes that we look for when using the TMJ arthroscopy as treatment modality for lysis and lavage are the outcomes that are commonly measured in patients with ID of TMJ using validated scales either for pain levels or the jaw range of motion. For diagnostic purposes, we will look at the potential of TMJ arthroscopy to assess the disc position and reducibility as well as its ability to detect adhesions and perforations.

\section{Screening and Data Extraction}

The selection of the studies will initially involve screening the titles and abstracts according to the inclusion and exclusion criteria and will be conducted by two investigators (WA and NA). Irrelevant studies such as reviews and not related to TMJ disorders will be removed from the list. We will then obtain the full-text articles for the list of potential eligible studies through various sources. These include full-text online journals and hardcopies available from the library. Articles which are not available from these two sources will be obtained through inter-library loans.

The full paper screening will then be reviewed by 3 investigators (WA, JH and FR). Any disagreements will be rectified through discussion and the article will be re-evaluated before the final agreement will be taken following consensus.

For the purpose of data extraction, we will create a data abstraction form using Microsoft Excel according to the study objectives. A pilot data collection will be carried out on five randomly selected studies included in the review to ensure practicality and suitability of the form. If necessary, we will make adjustment of the data extracted form before fully utilised. The data extraction again will be carried out independently by at least two of the investigators. The items included in the data extraction form for diagnostic studies are: author's name, year of publication, country, study type, patients, joints, diagnosis, disc position, disc reducibility, adhesions, perforations, agreement on diagnosis and percentage of agreement between MRI and arthroscopy (Table-2). Whereas the items tailored for therapeutic studies extraction form are: author's name, year of publication, country, study type, patients, joints, preoperative and postoperative records of pain and mouth opening, follow up and complications (Table-3).

Table-2: Data Extraction Form (Diagnostic)

\begin{tabular}{|c|c|c|c|c|c|c|c|c|c|c|c|c|}
\hline 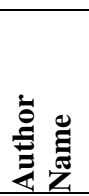 & 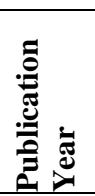 & $\begin{array}{l}\stackrel{E}{E} \\
\dot{\Xi}\end{array}$ & 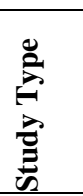 & : & $\stackrel{n}{\stackrel{0}{0}}$ & 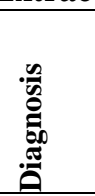 & 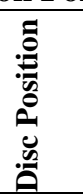 & 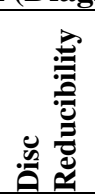 & 量 & 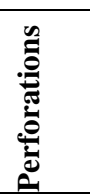 & 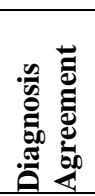 & 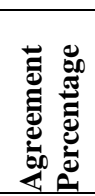 \\
\hline $\mathrm{XXX}$ & XXX & $\mathrm{XXX}$ & XXX & XXX & XXX & XXX & XXX & XXX & XXX & XXX & XXX & XXX \\
\hline
\end{tabular}

Table-3: Data Extraction Form (Therapeutic)

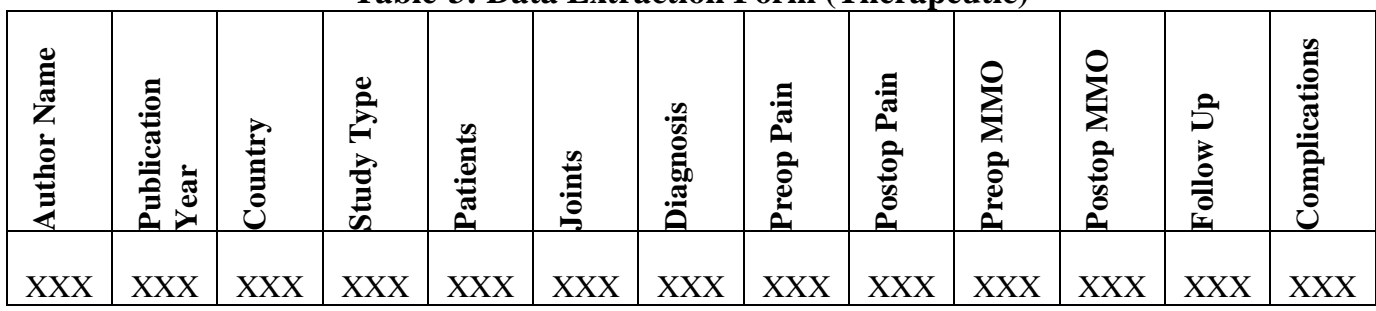

N.B: Preop $=$ Preoperative. Postop $=$ Postoperative

\section{Quality Assessment}

\section{For Therapeutic Randomized Studies}

Cochrane's collaboration tool for assessing risk of bias will be used. 5 domains are used to measure the quality of each study: sequence of randomisation, allocation concealment, blinding, incomplete data and selective reporting (also includes poor reporting). Risk of bias will be determined as a low, high or unclear based on guidance in Huggins et al., [21].

\section{For Therapeutic Nonrandomised Studies}

Newcastle Ottawa Scale (NOS) will be used. It was developed by Wells et al., to assess the quality of nonrandomised studies [22]. The NOS assigns up to a maximum of nine points in three domains: 1) selection of study groups (four points); 2) comparability of groups (two points); and 3) ascertainment of exposure and outcomes (three points) for case-control and cohort studies, respectively [23]. NOS final score will then be 
converted to the Agency for Healthcare Research and Quality (AHRQ) standards of Good, Fair, or Poor [24].

\section{For Diagnostic Studies}

Quality Assessment of Diagnostic Accuracy Studies revised tool (QUADAS-2) for the quality assessment of diagnostic accuracy studies will be used to assess the risk of bias. 4 domains are used to measure the quality of each study: patient selection, index test, standard reference and flow/timing. Risk of bias will be determined as a low, high or unclear based on guidance in Withing et al [25].

\section{Data Synthesis}

We will conduct a narrative synthesis for all studies included and this will include a narrative report of the risk of bias. We will use graphs to illustrate risk of bias of the included studies.

In case meta-analysis has been conducted, we will calculate the effect size (ES) for the studies included. The effect size will be calculated using the standardised mean difference (SMD) using Hedges, d [25]. By standardising all the mean available, it will ignore the variability of scales that have been used in the primary studies and transformed it to a common mean, hence allowing combination of various effect sizes. We will measure the ES for preoperative and postoperative records of the intervention group as well as the controlled group as appropriate for comparison. The ES from the individual studies will be pooled using the random effects model with the assumption that data will be heterogenous in view of variances in the study characteristics [26].

The results of the meta-analysis will be reported with $95 \%$ confidence interval and the $\mathrm{z}$ statistic will be used to test the significance of the ES. The ES will be interpreted as such (0.20: small effect, 0.50 : moderate effect and 0.80: large effect) [27]. We will present the ES using the Forest plot. Presence of publication bias will be examined visually through the generation of funnel plot and statistically with Egger's regression asymmetry test. All statistical analysis and graphical presentation will be performed using RevMan statistical software.

\section{DISSEMINATION}

One of the claimed advantages of this study is the used search strategy which will supposedly detect all related published studies to the subject under research and will allow less possibility of duplication and irrelevancy. In addition of avoiding missing out any potential source of information. Also, clarity and reproducibility of the protocol proposed to conduct this review systematically will provide more sophisticated way to investigate the subject of interest.

Regarding limitations of this study, they include the heterogeneity of studies (randomised and non-randomised) that will be included which may decrease the statistical power of the study and increase its risk of bias. Additionally, is the scarcity of studies investigating of the diagnostic potential of TMJ arthroscopy in the field of oral and maxillofacial surgery in comparison to other fields such as orthopaedics.

This review will try to demonstrate the capability of ALL to have favourable effect on the improvement of the symptoms of ID of TMJ. In addition, it will also verify the potential of TMJ arthroscopy as reliable diagnostic tool to provide final diagnosis of ID of TMJ. Such observations will be of relevance to the future clinical practice in terms of providing the best option to the patient as the use of arthroscopy as a diagnostic and therapeutic tool for management of TMD is uncommon, this may be due to of the lack of training courses integrated in classic training programs that led to unfamiliarity with its potentials, benefits and techniques.

\section{REFERENCE}

1. Efeogluet, C., Calis, A. C., Koca, H., \& Yuksel, E. (2018). A stepped approach for the management of symptomatic internal derangement of the temporomandibular joint. Journal of Otolaryngology - Head and Neck Surgery, 47(1), 33-40.

2. Yang, Ch., Zheng, J., \& Liu, X. (2017). Temporomandibular joint disc repositioning with arthroscopy: part III -detailed introduction of the technique. Almanac of Clinical Medicine, 45(6):460-465

3. Ulmner, M., Kruger-Weiner, C., \& Lund, B. (2017). Patient specific factors predicting outcome of temporomandibular joint arthroscopy: a 6-year retrospective study. Journal of Oral and Maxillofacial Surgery, 8(75), 1643-1647.

4. García, R. G. (2015). The Current Role and the Future of Minimally Invasive Temporomandibular Joint surgery. Oral and Maxillofacial Surgery Clinics of North America, 27(1):69-84.

5. Sato, F.R. L., Lima, C. A. A., Tralli, G., \& Da Silva, R. A. (2018). Is there a correlation between arthroscopic findings and the clinical signs and symptoms of patients with internal derangement of the temporomandibular joint? A prospective study. International Journal of Oral and Maxillofacial Surgery, 48(2):233-238.

6. Seebauer, C., Kaduk, W.J., Sanroman, F., \& Silva G. R. (2019). Temporomandibular surgery: surgical arthroscopy. In Connelly S. T., Tartaglia, G. M., \& Silva, G. R (Eds), Contemporary Management of Temporomandibular Disorders: Surgical Treatment (P. 78). Cham, Switzerland: Springer.

7. Abboud, W. A., Givol, N., \& Yahalom, R. (2015) Arthroscopic lysis and lavage for internal derangement of the temporomandibular joint. Annals of Maxillofacial Surgery, 5(2):158-162. 
8. Amin, M. F., Hassan, A. M., \& Barakat, K. I. (2012). The accuracy of dynamic Magnetic Resonance Imaging in evaluation of internal derangement of the temporomandibular joint; comparison with arthroscopic findings. The Egyptian Journal of Radiology and Nuclear Medicine, 43(3):429-436.

9. Alarabawy, R. A., El Ahwal, H. M., El Sergany, M. A., \& Mehrez, W. W. (2016). Magnetic resonance imaging evaluation of temporomandibular joint disorders, criterial analysis and significance in comparison with arthroscopy. The Egyptian Journal of Radiology and Nuclear Medicine, 47(2):467-475.

10. Hossameldin, R. H., \& McCain, J. P. (2016). Arthroscopy. In Tamimi, D., \& Hatcher, D. C (Eds), Specialty imaging: temporomandibular joint (PP. 358). Amsterdam, Netherlands: Elsevier

11. Rab, M. A., Chowdhury, G. M., Al-Mehedi, A., Sindhi, Q., Kabir, M. I., \& Sharmin. S. (2016). Management of Temporomandibular Joint Disorders by Arthroscopic Lavage. Journal of Armed Forces Medical College, Bangladesh, 12(1):88-93.

12. Tozoglu, S., Al-Belasy, F. A., \& Dolwick, M. F. (2011). A review of techniques of lysis and lavage of the TMJ. British Journal of Oral and Maxillofacial Surgery, 49(4):302-309.

13. McCain, J. P., Rua, H., \& Le Blanc, W. G. (1991). Puncture technique and portals of entry for diagnostic and operative arthroscopy of the temporomandibular joint. Arthroscopy: The Journal of Arthroscopic \& Related Surgery, 7(2):221-232.

14. McCain, J. P (1996). Puncture techniques and arthroscopic anatomy. In McCain JP (Ed), Principles and practice of temporomandibular joint arthroscopy (PP. 151-155). Missouri, United States: Mosby.

15. McCain, J. P., La Rua, H. D., \& Le Blanc, W. G. (1989). Correlation of clinical, radiographic, and arthroscopic findings in internal derangements of the TMJ. Journal of Oral and Maxillofacial Surgery, 47(9):913-921.

16. Higgins, J. P. T., Thomas, J., Chandler, J., Cumpston, M., Li, T., Page, M. J., \& Welch, V. A (Eds). (2019). Cochrane Handbook for Systematic Reviews of Interventions version 6.0. The Cochrane Collaboration.

17. Moher, D., Liberati, A., Tetzlaff, J., \& Altman, D. G. (2009). Preferred Reporting Items for Systematic Reviews and Meta-Analyses: The PRISMA Statement. British Medical Journal, 339, 2535.

18. Brown, P., Brunnhuber, K., Chalkidou, K., Chalmers, I., Clarke, M., Fenton, M., Forbes, C., Glanville, J., Hicks, N. J., Moody, J., Twaddle, S., Timimi, H., \& Young, P. (2006). How to formulate research recommendations. British Medical Journal, 333(7572), 804-6.
19. Scott, R. W., Wilson, M. C., Nishikawa, J., \& Hayward, R. S. A. (1995). The Well-built Clinical Question: A Key to Evidence-based Decisions. ACP Journal Club, 123(3), 3-12.

20. Lefebvre, C., Manheimer, E., \& Glanville, J. (2011). Searching for studies. In Higgins, J., \& Green, $S$ (Eds). Cochrane Handbook for Systematic Reviews of Interventions. Version 5.1.0 (updated March 2011). The Cochrane Collaboration.

21. Higgins, J. P. T., Savović, J., Page, M. J., Elbers, R. G., \& Sterne, J. A. C. (2019). Assessing risk of bias in a randomized trial. In Higgins, J. P. T., Thomas, J., Chandler, J., Cumpston, M., Li, T., Page, M. J., \& Welch, V. A. (Eds). Cochrane Handbook for Systematic Reviews of Interventions version 6.0. The Cochrane Collaboration.

22. Wells, G. A., Shea, B., O'Connell, D., Peterson. J., Welch, V., \& Losos, M (Eds). (2009). The Newcastle-Ottawa Scale (NOS) for assessing the quality if nonrandomized studies in meta-analyses. Retrieved Oct 10, 2019, from http://www.ohri.ca/programs/clinical_epidemiolog y/oxford.htm

23. Lo, C. K. L., Mertz, D., \& Loeb, M. (2014). Newcastle-Ottawa Scale: comparing reviewers` to authors' assessments. Medical Research Methodology, 14(1), 45.

24. Song, J., Tark, A., \& Larson, E. L. (2019). The relationship between pocket hematoma and risk of wound infection among patients with a cardiovascular implantable electronic device: An integrative review. Heart \& Lung. 49(1):92-98. Also available as a supplement from: https://www.ncbi.nlm.nih.gov/books/NBK115843/ bin/appe-fm3.pdf

25. Whiting, P. F., Rutjes A. W. S., Westwood, M. E., Mallett, S., Deeks, J. J., Reitsma, J. B., Leeflang, M. M. G., Sterne, J. A. C., Bossuyt, P. M. M., \& the QUADAS-2 Group. (2011). QUADAS-2: a revised tool for the quality assessment of diagnostic accuracy studies. Annals of Internal Medicine, 155(8), 529-36.

26. Borenstein, M., Hedges, L. V., Higgins, J. P. T., \& Rothstein, H. R. (2009). Effect Sizes Based on Means. In Borenstein, M., Hedges, L. V., Higgins, J. P. T., \& Rothstein, H. R (Eds), Introduction to Meta Analysis (PP. 21-32). Chichester, England: John Wiley \& Sons Ltd.

27. Borenstein, M., Hedges, L. V., Higgins, J. P. T., \& Rothstein, H. R (Eds). (2009). Random Effect Models. In Borenstein, M., Hedges, L. V., Higgins, J. P. T., \& Rothstein, H. R (Eds), Introduction to Meta- Analysis (PP. 69-75). Chichester, England: John Wiley \& Sons Ltd.

28. Cohen, J (Ed). (1988). Statistical power analysis for the behavioural sciences. USA, Mahwa: Lawrence Earlbaum Associates. 